\title{
perifèria
}

Número 5, Diciembre 2006

www. periferia.name

\section{Historias de vida y Ciencias Sociales. Entrevista a Franco Ferrarotti}

\author{
Montserrat Iniesta ${ }^{1}$ (Museu de Vilafranca/ICA) y Carles Feixa (UdL) ${ }^{2}$
}

\section{Resumen}

A La autonomía del método biográfico, las historias de vida como praxis interactiva, el carácter participativo de la investigación con fuentes orales, la propuesta de leer una sociedad a través de una biografía, son principios epistemológicos cuyos fundamentos teóricos deben bastante a las aportaciones del sociólogo italiano Franco Ferrarotti. Catedrático en la Universidad de Roma "La Sapienza", el autor se inscribe en la rica tradición de la escuela italiana de historia oral, que desde Gramsci, De Martino y Bosio ha utilizado las autobiografías como un acceso privilegiado a las clases subalternas. Sus libros Storia e storie di vita (1981) y La storia e il quotidiano (1985) constituyen obras fundamentales para los que trabajan con fuentes orales. La presente entrevista recoge los planteamientos del autor sobre la relación entre historias de vida y ciencias sociales.

\begin{abstract}
The autonomy of the biographical method, the interactive praxis of life stories, the participative character of the research based on oral sources, the proposal of reading a society through biographies, are all epistemological principles which theoretical foundations are in debt with the contributions of I talian sociologist Franco Ferrarotti. Chair of the Università di Roma "La Sapienza", the author belongs to the rich tradition of Italian school of oral history, that from Gramsci, De Martino and Bosio has used the autobiographies as a privileged acces to the subaltern classes. His books Storia e storie di vita (1981) and La storia e il quotidiano (1985) are basic works for researchers using oral sources. The present interview gathers the author's ideas about the relationship between life stories and social sciences.
\end{abstract}

\section{I ntroducción}

\footnotetext{
${ }^{1}$ Montserrat Iniesta (Barcelona, 1961) es antropóloga y directora del Museu de Vilafranca. Se ha especializado en el estudio antropológico del patrimonio, realizando estancias en centros museísticos de Francia, Italia, México y Canadá. Conservadora Internacional del Patrimonio por la Ecole nationale du patrimoine, es autora del proyecto museológico del Museu de l'Aigua (Lleida), Musée de Cerdagne (Francia) y Museu del Vi (Vilafranca del Penedès). Ha publicado Menges del Alt Urgell (1991), 'Entre bosc i riu hi passavem la vida'. El temps dels rais (1992), Els gabinets del món. Antropologia, museus i museologies (1994), así como diversos artículos en publicaciones como L'Avenç, Nos/Otros, I giorni cantati, etc. Forma parte del consejo editorial de las revistas Dyonisos y Revista d'Etnologia de Catalunya.

2 Carles Feixa (Lleida, 1962) es antropólogo y profesor en la Universitat de Lleida. Se ha especializado en el estudio antropológico de las culturas juveniles. Doctor por la Universidad de Barcelona, ha sido investigador visitante en centros académicos de México, París, California en Berkeley y Roma. Ha publicado La tribu juvenil (1988); El temps dels rais (1992), De jóvenes, bandas y tribus (1998); El reloj de arena (1998), Grafitis, grifotas, okupas (2002), entre otros libros y artículos. Forma parte del consejo editorial de las revistas Nueva Antropología (México), Nómadas (Bogotá), Young (Helsinki) y Revista d'Etnologia de Catalunya.
} 


\section{perifèria}

Número 5, Diciembre 2005

www. periferia. name

A principios de 1981, poco después de la aparición de Vite di periferia, Gérard Lutte, profesor de psicología en la Universidad de Roma-La Sapienza, hacía el siguiente comentario en la presentación del libro de su amigo Franco Ferrarotti: “Leyendo estas historias me he dado cuenta de que aportan información no sólo sobre el entrevistado, sino también sobre el entrevistador, sobre la manera de dirigirse a los demás, sobre su capacidad para identificarse con una situación de marginalidad, sobre el conocimiento que tiene de la situación en la que indaga, sobre la pertinencia de las preguntas formuladas. El método de las historias de vida es extremadamente sincero -y peligroso- porque nos obliga a descubrirnos, porque no permite esconderse tras el pretendido cientifismo y neutralidad de instrumentos considerados objetivos. Revela que la investigación sociológica y psicológica es una relación entre personas" (Lutte 1981). Eran observaciones muy pertinentes que servían de presentación a una investigación empírica sobre la pobreza en Roma del equipo dirigido por Ferrarotti, cuyo fundamento teórico y metodológico era un libro de referencia que aquel mismo año había publicado el autor: Storia i storie di vita (1981). Este lúcido ensayo -que desgraciadamente no ha sido traducido al castellano y que ha llegado en su versión francesa- entroncaba con la propia trayectoria del autor y con la fecunda tradición científica italiana que desde los años 50 ha venido utilizando las fuentes orales como una forma de investigación participativa y militante contra el idealismo de las ciencias sociales. ${ }^{3}$

Franco Ferrarotti, titular de la cátedra de sociología en la Università degli Studi di Roma-La Sapienza, es uno de los científicos sociales italianos más prestigiosos. Nacido en Piemonte en 1926, estudió en Turín, Londres y Chicago. Como investigador comprometido, participó en la fundación del Consejo de Poderes Locales de Europa en Ginebra en 1949 y trabajó como director de proyectos sociales de la OCSE en París en 1958. En 1959 obtuvo la primera plaza de sociología que se concedió en la universidad italiana. Diputado independiente en el Parlamento de la tercera legislatura, se retiró de la vida política para dedicarse de lleno al estudio y la investigación. Ha sido fundador y director de La Critica

\footnotetext{
${ }^{3}$ Esta entrevista fue realizada en Roma en diciembre de 1986, durante una estancia de corta duración financiada por la CIRIT. Diversos avatares han retrasado desde entonces su publicación. A pesar del tiempo trascurrido, creemos que su contenido no ha perdido vigencia, por lo que hemos creído oportuno recuperarla. La trascripción y traducción de la conversación oral son obra de los autores del texto.
} 


\section{perifèria}

Número 5, Diciembre 2005

www. periferia. name

Sociologica, una de las más prestigiosas revistas italianas de ciencias sociales. Persona de gran simpatía y buen conocedor de la realidad española -durante el franquismo hizo en alguna ocasión de enlace entre comunistas italianos y catalanes-, Ferrarotti ha destacado por su defensa de una visión humanística de las ciencias sociales en general y del método biográfico en particular. Sobre estos temas, a los que ha dedicado numerosos artículos y publicaciones (vid. infra), mantuvimos con él una enriquecedora conversación. ${ }^{4}$

\section{La aproximación autobiográfica}

- Al llegar a Italia, nos ha sorprendido el creciente interés que, en el mundo académico, suscita el método biográfico. En las librerías se encuentra gran cantidad de publicaciones y un número considerable de simposios y coloquios abordan el tema. ${ }^{5}$ ¿Qué opinión le merece este fenómeno?

Bien, en primer lugar os diré que estoy de acuerdo en que hay un interés por las historias de vida, por la aproximación autobiográfica, pero desgraciadamente no creo que, hoy por hoy, este interés sea mayoritario. La mayoría de investigaciones realizadas en Italia en este momento en el campo de las ciencias humanas (psicología social, antropología, sociología...) todavía se basan en métodos y técnicas cuantitativas, sobre todo el sondeo centrado en una muestra más o menos representativa. Se trata de investigaciones financiadas por entes públicos y privados -empresas, ayuntamientos, sindicatos, etc.-, que obtienen de este modo resultados más fácilmente presentables como seguros. A los administradores de las

\footnotetext{
${ }^{4}$ Durante los años 50, cuando Ferrarotti empezó a utilizar las historias de vida en sus investigaciones, se había comenzado a publicar diversos volúmenes de los Quaderni del carcere, en los que Antonio Gramsci reivindicaba el papel de las autobiografías para reconstruir una historia nacional-popular. "In Italia mancano i memorialisti e sono rari i biografi e gli autobiografi. Manca l'interessse per l'uomo vivente, per la storia vissuta" (Gramsci 1971: 91). Por aquellos mismos años, Ernesto de Martino (1961) utilizaba las fuentes orales como vía primordial para estudiar las culturas subalternas de la Italia meridional; intelectuales comprometidos como Gianni Bosio (1963) proclamaban el "elogio del magnetófono" como base para la emergencia de una nueva cultura popular y proletaria (que siempre había sido subalterna en relación con las fuentes escritas); poetas como Pier Paolo Pasolini (1955) utilizaban los relatos orales como estrategia para conocer las formas de vida emergentes en la periferia urbana. Eran esfuerzos para "dar voz, hacer hablar a los otros", reaccionando contra el idealismo dominante en literatura, el cuantitativismo dominante en sociología y la Applied Anthropology de origen norteamericano, que tras la II Guerra Mundial tomó la Italia meridional como laboratorio de investigación privilegiado (Cfr. Feixa 1987-88, 1996).

${ }^{5} \mathrm{La}$ actualidad de las fuentes orales en la Italia de los 80 se puso de manifiesto en coloquios como Biografia, storia e società (Macioti 1985); Fonti orali: fra antropologia e storia (Bernardi et al. 1977); revistas como Fonti Orali, I giorni cantati; instituciones como I'Istituto Ernesto de Martino, el Circolo Bosio, etc. Conviene destacar, como precedentes, las aportaciones de autores como Montaldi (1961), Revelli (1977), Passerini (1974), Cavallaro (1981), De Angelis (1981), Zuucconi (1984), Portelli (1985), entre otros. Sobre la escuela italiana de historia oral, véase Feixa 1988, Contini 1991 y Portelli 1992.
} 


\section{perifèria}

Número 5, Diciembre 2005

www. periferia. name

grandes empresas les gustan sobre todo los resultados que se pueden presentar en tablas, el aparato estadístico, los porcentajes, las correlaciones, la medición de los fenómenos. Dicho esto, estoy de acuerdo en que no se puede negar que hay en I talia una consciencia creciente del carácter ilusorio de estas mediciones, es decir, el carácter ilusorio de la seguridad que dan las investigaciones cuantitativas, que miden los fenómenos con gran minuciosidad estadístico-matemática, pero que son limitadas desde el punto de vista de la interpretación.

Por eso no ha de extrañarnos que, sobre todo para algunos problemas, el interés por la aproximación autobiográfica esté aumentando. Y ello por dos razones. La primera es que a menudo los problemas sociales más graves de una sociedad son problemas clandestinos que no se pueden cuantificar. La segunda, porque ciertos colectivos sociales importantes, como por ejemplo los toxicómanos, no son representativos desde un punto de vista estadístico. Y sólo se pueden estudiar, como hemos hecho nosotros, intentando establecer con ellos una relación de confianza, lo que no ofrecen las investigaciones tradicionales, que no consideran esta relación necesaria. De hecho, mi libro Giovani e droga (1977), que se basa en 49 entrevistas con toxicómanos, es hasta ahora uno de los pocos estudios realizados con historias de vida sobre la droga. $Y$ es que, en el fondo, lo que se suele hacer, más que investigaciones sobre la droga, son estudios sobre la visión del problema de la droga entre los no drogadictos, o sobre los toxicómanos en tratamiento, que son a los que se puede aplicar una encuesta. $Y$ sin embargo, con estos métodos no se consigue nunca llegar a comprender por qué han empezado a drogarse. ¡Y no digamos el terrorismo! De hecho, yo me acabé de convencer de la fecundidad del método autobiográfico al ocuparme precisamente del terrorismo, porque con este fenómeno no puede utilizarse otro método sino el de la confesión, que implica una relación de confianza con el terrorista.

- Hay que decir, sin embargo, que usted no siempre ha mantenido esta postura respecto a los métodos cualitativos, hacia los cuales mostraba una cierta desconfianza en sus primeras investigaciones. ¿Cuáles son los motivos que le hicieron optar por la aproximación autobiográfica como eje fundamental de sus estudios?

Es cierto. Hasta 1959, cuando publiqué La piccola città (es decir, Castellamare di Stabia), fruto de una investigación sobre sociología industrial, no me di cuenta de que nuestra investigación, centrada en fenómenos cuantitativos sobre el interior de la fábrica, no tenía sentido si no se tomaba en consideración las actitudes de las personas fuera de la fábrica. Pero estas actitudes sólo se podían entender mediante una relación directa, biográfica. A menudo me he parado a pensar por qué escogí 


\section{perifèria}

Número 5, Diciembre 2005

www. periferia. name

los métodos cualitativos en vez de los cuantitativos, que tienen más financiación, rinden más y dan más poder. Y sólo puedo decir lo siguiente: mi opción por los métodos cualitativos es paralela, o al menos comparable, a mi opción por la sociología cuando en Italia la sociología no existía. En 1942-43, en plena guerra, me acababa de matricular en la universidad. Como no gozaba de muy buena salud, me mandaron a la costa occidental de Liguria. Tenía una grave enfermedad pulmonar y disponía de mucho tiempo, que pasaba leyendo. La biblioteca de San Remo, sin embargo, hacía tiempo que no adquiría libros. Todos lo que había eran de principios de siglo. Eran los famosos libros de los hermanos Bocca, editores de Turín, de línea digamos positivista, una sociología de finales del XIX. Había sociólogos como Morasso, Sigele, Pareto... El hecho es que no leí nada de Croce y demás. Y aunque esto suene mal, he de estar muy agradecido a la mala política de adquisiciones de las bibliotecas municipales italianas por haber podido leer libros que nadia leía. Para decirlo sintéticamente, buscaba algo menos abstracto que la filosofía hasta entonces dominante en Italia, que era la filosofía idealista crociana, neo-hegeliana, y al mismo tiempo algo no tan árido como la economía política. Me interesaba por la filosofía y por la economía el mismo tiempo. Pero la economía política era de tipo matemático-cuantitativo, de estilo muy abstracto - utilidad marginal, valor, etc.-, mientras que la filosofía era metafísico-dialéctica, pero en un sentido racionalista reductivista. Yo buscaba un término medio, algo conceptual, pero al mismo tiempo empírico. Y por eso escogí la sociología. El problema es que entonces no existía la sociología. Por eso no había pensado nunca en convertirme en profesor universitario. De hecho, cuando acabó la guerra me fui a estudiar al extranjero, primero a Francia, después a Inglaterra y finalmente a los Estados Unidos.

\section{El sueño de Escipión}

- De su estancia en los Estados Unidos ha hablado en su obra La storia e il quotidiano (1986), en la que constata el fracaso de su particular "sueño de Escipión". ¿Qué significado tiene esta expresión?

El sueño de Escipión es un fragmento muy bello de Cicerón, en el que el viejo Escipión Africano habla a su sobrino Emiliano y le explica cuál sería la vida ideal: consistiría en una unión de la vida teórico-especulativa griega -el teoréticos-biosunida con el espíritu práctico, constructivo de los romanos. Cuando fui a los Estados Unidos en 1950, había traducido ya a Thorsten Veblen (La teoría de la clase ociosa) y precisamente fui para ver dónde había nacido y enseñado Veblen: a Chicago. Mi intención era equiparable al sueño de Escipión: por un lado, aprender las técnicas empíricas de los americanos, que eran bastante sofisticadas, y unirlas con la gran 


\section{perifèria}

Número 5, Diciembre 2005

www. periferia. name

tradición del pensamiento social clásico europeo: Marx, Weber, Pareto, Durkheim, Ortega, que había leído cuando era muy joven y me impresionó mucho -El amanecer de la razón histórica, El tema de nuestro tiempo, la idea de generación...-, debo mucho a Ortega. Pero no resultó. Falló. No sé porqué. Todavía pienso en ello. Creo que en aquello que yo consideraba una cuestión técnica había implícitos ciertos valores. Las técnicas de investigación empírica no son sólo técnicas, tienen un aspecto meta-técnico. Cuando aplico empíricamente una de estas técnicas, ya estoy formalizando, deificando los seres humanos a los que me dirijo, porque preveo las respuestas que me darán. $Y$ desde el momento que preveo su comportamiento, ya he limitado su humanidad, que es imprevisible y no es problemática. La síntesis entre las técnicas americanas de investigación empírica y la tradición del pensamiento europeo no era una síntesis, era una confrontación. Había sólo una vía para recuperar críticamente ciertos instrumentos de análisis y era precisamente la de las historias de vida. Las historias de vida respetan el momento imprevisible del comportamiento: se acepta a la persona como tal, no se la mediatiza para hacerla entrar en las casillas del cuestionario. Después, una vez se ha expresado, viene el momento interpretativo, propiamente hermenéutico, en el que puedo hacer intervenir parámetros de catalogación relativos. De esta manera consigo ligar teoría e investigación, hacer convivir el elemento empírico y el teórico. Pero, naturalmente, dejando siempre abierto un gran espacio para lo imprevisible, para el momento problemático, para aquello no exactamente definible a priori. Y esta es mi postura actual, una postura muy poco cómoda, pero es la única que no intenta confirmar los prejuicios apriorísticos del investigador.

- En 1959 ganó el primer concurso para una plaza de sociología que convocaba la universidad italiana. ¿Cuál era la situación de las ciencias sociales italianas en los años 50, a su regreso de su experiencia americana?

Cuando volví, I talia estaba en plena industrialización. Y según mi argumento teórico, Italia, como todos los países que se industrializan, debía dotarse de un instrumento de autoescucha y autodirección racional, porque la modernidad significa la crisis de la tradición como fuente de legitimidad sustancial de las decisiones sociales. La única alternativa a la tradición era para mí el cálculo racional a través de la autointerrogación, es decir, el análisis sociológico. Creía mucho en esto. Todo el mundo me decía: ¿por qué no haces filosofía, así podrás empezar la vida universitaria? Y yo respondía que no contasen conmigo, que yo sólo estaba disponible para la sociología. ¡Y paradójicamente la sociología llegó a Italia! En 1959 se convocó el primer concurso para una plaza de sociología en la universidad italiana y, naturalmente, lo gané yo porque era el único. El hecho es que no había considerado nunca la posibilidad de dedicarme a la vida académica. Al contrario, 


\section{perifèria}

Número 5, Diciembre 2005

www. periferia.name

consideraba la sociología como una ciencia no apta para limitarse al mundo académico, sino que veía necesaria una intervención en el mundo.

\section{La investigación social como participación}

- Esta vocación de intervención social ¿no se vincula de alguna manera a su propuesta de la sociología como participación, que defiende en su libro Una sociologia alternativa (1972), y la noción más reciente de biografía como praxis interactiva?

Sí, pero es algo diferente. Precisamente cuando regresé a Italia en 1953 me di cuenta de que la forma de hacer sociología que había aprendido en América, la sociología empírica, positivista, no me convenía. Porque la verdadera diferencia entre la sociología y las ciencias naturales no reside en el método, sino en el hecho de que el objeto coincida con el sujeto. Si los sociólogos adoptasen las técnicas de las ciencias naturales, en realidad estarían confirmando la asimetría entre el investigador y el objeto de la investigación. En otras palabras, los sociólogos trataban los objetos de investigación, que eran personas, como si fueses cosas. Por lo tanto, había un elemento terriblemente autoritario en el modelo de investigación, que me molestaba mucho. ¿Por qué se hacía esto? Porque el mercado lo reclamaba. La sociología era en el fondo una ciencia instrumental subordinada al mercado, que se vendía al mejor postor. Entonces concebí la idea de que había un círculo hermenéutico que convertía a todo investigador en investigado. Por ejemplo, en el momento que interrogamos a otro sobre la clase social, no podemos hacerlo sin un presupuesto, y el presupuesto en ciencias humanas es el punto de vista del investigador, lo que llamo "la declaración preliminar", que no es solamente la declaración de valores, como decía Myrdal, es la autocolocación histórico-políticomoral. Esta autocolocación es el punto de vista que permite la perspectiva interpretativa del hecho social y coloca al mismo tiempo al investigado en un plano de paridad. De este modo, el investigador no sólo estudia al otro, sino que se estudia también a sí mismo. Buscando descubrir a los otros, acaba descubriéndose a sí mismo. Así pues, comprendí que fundamentalmente existían dos razones que explicaban la escasez de sociólogos que utilizasen las historias de vida, En primer lugar, el hecho de que los estudiosos no querían "descubrirse", que es siempre una operación muy peligrosa. En segundo lugar, querían tener encargos del mercado, disponer de fondos económicos y recursos. No es lo que a mí me interesaba. Poco a poco fui desarrollando mi trabajo sobre la base de la sociología como participación, sobre la base de los métodos cualitativos, que ya habían sido usados por los sociólogos. De hecho al principio fue como descubrir el Mediterráneo, pero de hecho la sociología positivista utilizaba y utiliza los métodos cualitativos, las historias de 


\section{perifèria}

Número 5, Diciembre 2005

www. periferia. name

vida, pero sólo con una función ilustrativa, secundaria, para añadir una pincelada de color humano.

Ahora mi posición se basa en la necesidad de invertir esta jerarquía. Habría que partir en primer lugar de las historias de vida, para que los problemas emerjan y se definan en las mismas palabras de los actores. En un segundo momento, una vez interpretados, quizás será posible vincularse en algunas ocasiones a parámetros empíricos, verificarse cuantitativamente. Pero ello no significa que los problemas fundamentales de las ciencias humanas sean verificables en un sentido cuantitativo. El problema humano indica siempre una tensión permanente, abierta, que se repropone continuamente en temas nuevos según las personas, los lugares, la época histórica. La vieja sociología se interesa por los problemas humanos, pero los pone al mismo nivel que los problemas técnicos. El problema de la sociedad, por tanto, se convierte en un problema de ingeniería social. Para mi, al contrario, los problemas de la sociedad van mucho más allá, no son solucionables de una vez para siempre, sólo se pueden entender en una perspectiva histórica. Los sociólogos positivistas, dicen que estudian la sociedad pero en realidad la deifican, es una sociedad bloqueada en una fórmula técnica, una sociedad que ya está muerta, congelada. Y para poder aceptar una sociedad sin matarla, hay que considerar el desafío de un objeto de estudio en continuo movimiento dinámico, hay que aceptar implicarse, estudiarlo a través de una empatía que sólo heurísticamente admite una distancia entre investigador e investigado. Por lo tanto, la paradoja actual de la sociología, de la sociología crítica, es que el sociólogo ha de ser al mismo tiempo analista y actor. Implicado, participante y capaz de una distancia crítica. Ello comporta muchos problemas porque entre otras cosas supone que no se puede estar a sueldo de nadie. Renunciar al mercado significa considerar la empresa sociológica como un compromiso de autoconciencia mediante la reflexión crítica de un proceso en el mismo momento en que este proceso se lleva a cabo. Para intentar esta vía, yo no he encontrado por el momento nada mejor que las historias de vida. 


\section{perifèria}

Número 5, Diciembre 2005

www. periferia.name

\section{La ciudad pequeña}

- La Piccola Città (1959) es la primera investigación en la que empieza a utilizar las historias de vida como estrategia para llegar a conocer la "ciudad oculta" que se esconde detrás de la estructura formal de las instituciones. Pero, si nos permite decirlo, el uso que hace del material autobiográfico es todavía instrumental, ilustrativo, subordinado a las hipótesis y a una descripción teórico-cualtitativa previa.

Sí, de hecho era una primera fase. En aquella época no estaba todavía convencido de lo que digo ahora. Ha sido un proceso bastante lento y difícil porque suponía separarse de la tradición en la que me había formado. Me daba cuenta entonces de que la mejor investigación cuantitativa tenía siempre un vacío porque faltaba el elemento humano. La piccola città deriva de otra investigación que estaba haciendo por encargo del IRP sobre las atarazanas de Catellamare di Stabia. Estas atarazanas estaban siendo reorganizadas por un ingeniero francés llamado Ravaille. En mi estudio, me di cuenta de que los principales obstáculos con los que topaba la reorganización no eran de naturaleza técnica, sino de naturaleza socio-psicológica, antropológica. Eran resistencias humanas al cambio. Yo estaba entonces trabajando en la universidad mediante una especie de encargo no retribuido y la empresa nos encargó estudiar dentro de la fábrica la causa de esta resistencia. Nosotros intentamos hacer un buen trabajo pero me di cuenta de que las resistencias reales procedían de fuera de la fábrica, de la comunidad. Pero a la empresa, la comunidad no le interesaba. El resultado fue que el dossier sobre la investigación, de más de mil páginas, nunca se publicó porque no había gustado a los dirigentes. El hecho es que para comprender estas cuestiones hacía falta estudiar primero el contexto, tener una idea del marco socio-económico y después tener las biografías de la gente. Pero es verdad que estas biografías tenían sólo una función ilustrativa. Sin embargo, daban algunos elementos cualitativos para comprender algunas cuestiones particulares. Por ejemplo: la figura del obrero que todavía es agricultor, que trabaja todavía "a destajo", en contacto con la práctica mafiosa; y después una modernización alejada de la racionalización capitalista, en la que prevalecen las prácticas familistas, hasta el punto que el trabajo a destajo se calcula no tanto sobre la base de lo que se trabaja, sino sobre la base de los amigos, de los parientes que se tiene, de la estructura jerárquica de la empresa. Es lo que alguien llamaría la "base moral de una sociedad atrasada". 


\section{perifèria}

Número 5, Diciembre 2005

www.periferia. name

- Esa fue la afirmación del antropólogo norteamericano Edward Banfield en un libro muy polémico que usted criticó. ${ }^{6}$ ¿En qué se basó su crítica a Banfield y cuál es su opinión sobre las relaciones entre antropología y sociología en Italia?

Mi crítica a Banfield es muy simple. Su error proviene de su etnocentrismo porque estudia la cultura tradicional de la Italia meridional a través de la óptica yanqui, hasta el punto de que llega a proponer como método para salir del familismo la elaboración de un semanario local, ¡sin darse cuenta de que gran parte de las personas eran analfabetas! Es decir, que proyecta las condiciones de Nueva Inglaterra sobre el campo italiano. Un pequeño error histórico-geográfico. Muchos investigadores americanos proyectaron y aplicaron sus sofisticadas técnicas a la realidad italiana muy escrupulosamente, pero sin preocuparse por analizar también los sistemas de vida estudiados, el contexto histórico y social, de comprender el significado de una situación histórica para los grupos humanos que la viven, situación que si se juzga partiendo de modelos externos, se corre el riesgo de aparecer simplemente sin sentido, absurda. Por otra parte, tenemos mucho contacto con los antropólogos italianos. Tanto en el Istituto di Sociología como en "La Critica Sociologica" , colaboramos con gente como Tullio Tentori, Vincenzo Padiglione, Renato Cavallaro, Vittorio Lanternari, etc, que son científicos muy valiosos. Es evidente que los antropólogos han utilizado a menudo las historias de vida en sus investigaciones y que éste ha sigo también un terreno de convergencia. Yo participé en el I Congreso de Antropología y he seguido teniendo contactos con los antropólogos.

\section{La autonomía del método autobiográfico}

- Hay una segunda fase en relación con su posición sobre el método biográfico, que corresponde a sus investigaciones sobre la marginalidad en Roma. ${ }^{7}$ En esta fase, los datos biográficos tienen ya una función cognoscitiva en sentido pleno, son el instrumento principal para hacer emerger los problemas y las hipótesis. Esta etapa converge en la propuesta teórica y metodológica de Storia e storie di vita (1981), libro que consiguió una fama notable, y que abrió un importante debate en el seno de las ciencias sociales italianas y también fuera del país. ${ }^{8}$ ¿Cuál fue la

\footnotetext{
${ }^{6}$ Banfield 1961; Ferrarotti 1966.

${ }^{7}$ Ferrarotti 1974, 1977, 1983.

8 "La historia de vida, lejos de proponerse como conjunto de elementos ilustrativos de lo ya conocido, complemento facultativo en clave cualitativa de los resultados "fuertes" de la investigación, adquiridos mediante las técnicas estandarizadas del la medida exacta, abre una fase nueva de la investigación en ciencias sociales (...) Cada historia de vida se revela incluso en sus aspectos menos generalizables como
} 


\section{perifèria}

Número 5, Diciembre 2005

www. periferia. name

evolución desde la primera etapa, en la que el uso de hacía del material biográfico era mucho más tímido y limitado?

Efectivamente, hay una primera fase en la que reconozco las limitaciones del método cuantitativo, pero no sé qué hacer con él. En una segunda fase empiezo a estudiar las historias de vida, los métodos cualitativos, pero los utilizo tímidamente, no me parecen suficientemente científicos, no quiero correr el riesgo de hacer literatura. Al fin y al cabo me doy cuenta de que existe una concepción plena de las historias de vida. Es decir, la posibilidad de utilizar las historias de vida como material científico fundamental a efectos de la interpretación. Debo decir que Storia e storie di vita es un texto polémico. Supone la ruptura total con los métodos cuantitativos y la afirmación de la autonomía del método biográfico, de su capacidad de desarrollo interno. La tesis central es que es posible leer una sociedad a través de una biografía. Por eso acabo este pequeño panfleto con la propuesta de estudiar no solo la biografía de un individuo, sino de centrarse en la biografía del grupo primario, porque el individuo no es nunca un individuo solitario, en el fondo es una síntesis, un signo cultural estenográfico. Es lo que llamo "el polo dialéctico de las biografías". Hay que tenir en cuenta el concepto de horizonte histórico, que significa, en primer lugar, el carácter no intemporal, no desarraigado de las relaciones materiales extra-subjetivas, de los documentos autobiográficos; y en segundo lugar, el conjunto de las relaciones estructurales, ya sean formalizadas en instituciones o bien expresadas en comportamientos y costumbres.

- En su libro La storia e il quotidiano (1986) incide en algunas reflexiones de tipo metodológico sobre la aproximación autobiográfica, al tiempo que critica el uso puramente literario de este material. ${ }^{9}$ ¿Puede hablarse de un "método" para la historias de vida?

Corremos el riesgo de pensar en las historias de vida como en un atajo, como una manera de ahorrarse el método. Es cierto que uno de los aspectos más fascinantes de la investigación con fuentes orales consiste en el hecho de que no existen unas reglas precisas, una especia de fórmula aplicable según las instrucciones de uso en

síntesis vertical de una historia social. Cada comportamiento o acto individual aparece en sus formas más únicas como síntesis horizontal de una estructura social... Nuestro sistema social se encuentra todo él en cada uno de nuestros actos, sueños, delirios, obras, comportamientos, y la historia de este sistema se encuentra toda ella en la historia de nuestra vida individual" (Ferrarotti 1981: 10 y 41).

9 "El intento (de este libro) es continuar y profundizar el discurso iniciado con Storia e storie di vita. Sobre todo en la tercera parte, el lector encontrará algunas reglas prácticas que convierten la aproximación biográfica científicamente productiva. Huelga decir que no se trata de instrucciones de uso, sino de reflexiones y métodos heurísticos madurados en el trabajo. El método que se fundamenta en las historias de vida no tolera los atajos mecanicistas que parecen tan eficaces para el análisis sociológico, quizás para hacer olvidar el tema y las razones de la investigación " (Ferrarotti 1986: 1). 


\section{perifèria}

Número 5, Diciembre 2005

www. periferia. name

cada caso examinado. El investigador que utiliza las historias de vida está constreñido a seguir el ejemplo de los clásicos, y a construir los instrumentos de investigación en el mismo hacerse de la investigación y en contacto directo con los problemas de los que ha decidido ocuparse y con los sujetos investigados. Pero ello no quiere decir se deba ignorar los problemas metodológicos y técnicos, o bien dejarlos al libre albedrío. Me he empezado a interesar por el hecho de que las historias de vida comportan un método, una vía de investigación, y además este método tiene unos momentos precisos. Se empieza evidentemente con la recogida del relato autobiográfico, que es fundamentalmente un acto de interacción; en el momento del análisis hay un momento de temporalización y uno de contextualización que es muy importante. Hay que tener en cuenta cuestiones como la observación participante y la convivialidad previa y posterior a la grabación de la historia, las reglas de la transcripción del material oral, el uso de fuentes complementarias para el análisis, etc. Estoy intentando desarrollar poco a poco estos momentos, pero es muy difícil. La storia e il quotidiano y mi siguiente libro Ricordo e temporalità son precisamente los primeros pasos en esta nueva dirección. ${ }^{10}$

\section{Bibliografía}

Banfield, E. 1961. Le basi morali di una società arretrata, Bologna, II Mulino.

Bernardi, B. et al. (eds). 1977. "Fonti orali: fra antropologia e storia“, Quaderni Storici, 35.

Bosio, G. 1963 (1975). “Elogio del magnetofono", in L'intellettuale rovesciato. Interventi e ricerche sulla emergenza d'interesse verso le forme di espressione e di organizzazione 'spontanee' nel mondo popolare e proletario, Milano, Bella Ciao.

Cavallaro, R. 1981. Storie senza storia. Una indagine sull'emigrazione calabrese in Gran Bretagna, Roma, Centro Studi Emigrazione.

\footnotetext{
${ }^{10} \mathrm{El}$ autor ha seguido publicando desde entonces trabajos sobre el método autobiográfico. En castellano debemos citar La historia y el cotidiano (1991) y diverersos artículos aparecidos en revistas como Historia, Antropología y Fuente Oral $(1998,1992,1993)$. Acaba de aparecer la traducción de uno de los últimos libros del autor, que supone el paso del estudio de la oralidad al estudio de la escritura (Leer, leerse, 2002). Por desgracia, no se ha traducido todavía su obra fundamental sobre el tema (Storia e storie di vita, 1981).
} 


\section{perifèria}

Número 5, Diciembre 2005

www.periferia. name

Contini, G. 1991. “La historia oral en Italia”, Historia y Fuente Oral, 5: 131-138.

De Martino, E. 1961 (1994) La terra del rimorso, Saggiatore, Milano.

De Angelis, R. 1981. Droga e controcultura nella peiferia urbana. Storie di vita della marginalità giovanile, Roma, Armando.

Feixa, C. 1987-88. "Més enllà d'Eboli. Gramsci i I'antropologia italiana", Nous Horitzons, Barcelona, 105: 28-41; 107: 21-32.

-- 1997. "De Martino dalla Catalogna", en C. Gallini; M. Masenzio (eds.), Ernesto de Martino nella cultura europea, Napoli, Liguori: 59-74.

Ferrarotti, F. 1959. Piccola città, Napoli, Liguori.

-- 1966. "Osservazioni intorno al rapporto tra antropologia e sociologia in Italia", De Homine, 17-18.

-- 1972. Una sociologia alternativa, Napoli, Liguori.

-- 1974. Vite di baraccatti, Napoli, Liguori.

-- 1977. Giovani e droga, Napoli, Liguori.

-- 1979. "Apppunti sul metodo biográfico", La critica sociológica, 47.

-- 1981. Storia e storie di vita, Bari, Laterza (Histoire et histoires de vie, Paris, Méridiens, 1983).

-- 1981b. Vite di periferia, Milano, Mondadori.

-- 1982. "Acerca de la autonomía del método biográfico", in J. Duvignaud (ed), Sociología del conocimiento, Méxuci, FCE.

-- 1986. La storia e il quotidiano, Bari, Laterza (1991. La historia y el cotidiano, Barcelona, Península).

-- 1987. Il ricordo e la temporalità, Bari, Laterza.

-- 1989. “Historia, biografía, privacy", Historia y Fuente Oral, 2: 51-56.

-- 1992. "Industrialización e historias de vida", Historia y Fuente Oral, 9: 163-186. 


\section{perifèria}

Número 5, Diciembre 2005

www. periferia. name

-- 1993. "Las biografías como instrumento analítico e interpretativo", en J.M.

Marinas; C. Santamaría (eds), La historia oral. Métodos y experiencias, Madrid, Debate.

-- 2002. Leer, leerse. La agonía del libro en el cambio de milenio, Barcelona, Península.

Gramsci, A. 1971. Letteratura e vita nazionale, Roma, Riuniti.

Lutte, G. 1981."A proposito di 'Vite di periferia'", La Critica Sociologica, 59.

Macioti, M.I. (ed). 1985. Biografia, storia e società, Napoli, Liguori.

Montaldi, D. 1961. Autobiografia della leggera, Torino, Einaudi.

Pasolini, P.P. 1955. Ragazzi di vita, Milano, Garzanti.

Padiglione, V. 1979. "Osservatore e osservato: problemi di conoscenza e interpretazione", Problemi del socialismo, XX, 15: 167-209.

Passerini, L. (ed). 1978. Storia orale, Torino, Rossenberg e Sellier.

Portelli, A. 1985. Biografia di una città. Terni, storia e racconto: 1830-1985, Torino, Einaudi.

-- 1992. “L"Elogio del magnetofono' alle origini della storia orale", II De Martino, 1: 29-35.

Revelli, N. 1977. II mondo dei vinti, Torino, Einaudi.

Zucconi, A. 1984. Autobiografia di un paese, Milano, Comunità. 